\title{
Statistical Methods in Endocrine Surgery Journal Club
}

\author{
${ }^{1}$ Prabhaker Mishra, ${ }^{2}$ Sabaretnam Mayilvaganan, ${ }^{3}$ Amit Agarwal
}

\begin{abstract}
Endocrine surgery is a relatively newer surgical subspecialty with few dedicated endocrine surgery departments and journals. To understand the published literature, knowledge of some common statistical methods are not only useful but also significant to publish own research successfully. This paper present a brief review of common statistical methods, used for data analysis in conducting medical research.
\end{abstract}

Keywords: Endocrine surgery, Statistical methods, Journal club, Normal data, Parametric test, Survival analysis, Regression analysis, ROC curve.

How to cite this article: Mishra P, Mayilvaganan S, Agarwal A. Statistical Methods in Endocrine Surgery Journal Club. World J Endoc Surg 2015;7(1):21-23.

Source of support: Nil

Conflict of interest: None

\section{INTRODUCTION}

Endocrine surgery is a relatively newer surgical subspecialty with few dedicated endocrine surgery departments and journals. Endocrine surgery represents a challenging field of uses with rich pathophysiological consequences and is a subject of never-ending research and also controversies. ${ }^{1}$ In modern clinical research, like other branches, this branch also has different up to date clinical surgical procedure and its outcome, that should be shared with other professionals with proper medium using evidence-based medicine. Published literature is one of the most appropriate medium among them. To understand the published literature, knowledge of some common statistical methods is not only useful but also to publish own research successfully. This paper presents a brief review of common statistical methods used for data analysis in conducting research.

In endocrine surgery, journal club is organized to discuss and critically evaluate the recent articles in the

\footnotetext{
${ }^{1,2}$ Assistant Professor, ${ }^{3}$ Professor

${ }^{1}$ Department of Biostatistics and Health Informatics, Sanjay Gandhi Post Graduate Institute of Medical Sciences, Lucknow Uttar Pradesh, India

${ }^{2,3}$ Department of Endocrine Surgery, Sanjay Gandhi Post Graduate Institute of Medical Sciences, Lucknow, Uttar Pradesh, India

Corresponding Author: Sabaretnam Mayilvaganan, Assistant Professor, Department of Endocrine Surgery, Sanjay Gandhi Post Graduate Institute of Medical Sciences, Lucknow, Uttar Pradesh, India, Phone: 919655851510, e-mail: drretnam@ gmail.com
}

field. For that purpose, statistical knowledge is important because some of these results might be contradictory or might have employed wrong statistical techniques. Endocrine surgeon can use these new advances in surgery for improving patient's care. These recent advances, methods, complexity and experiences can be shared by published literature by a way that all the procedure can be discussed in easy way, so that everyone can understand.

Statistics is a way to summarizing, presenting, describing and interpreting data in medical practices, which is useful to publish good scientific research paper. In the present paper, many common statistical methods have been discussed which are frequently used in medical literature and research to present the outcome of study.

\section{DISCUSSION OF COMMON STATISTICAL METHODS}

The proper understanding and use of statistical tools are essential in medical research. There are certain assumptions for statistical tests that must be satisfied before to use. Data are important factor to decide about statistical test. ${ }^{2}$ For quantitative and qualitative data, types of statistical techniques are different. Also for same type of data, statistical techniques are different depending upon certain conditions. In medical statistics, descriptive statistics, parametric and nonparametric test, association, correlation and regression, survival analysis and accuracy of the diagnostic test are frequently used in medical research. ${ }^{2-4}$

When data are continuous, and objective is to describe the data in statistical measures, descriptive statistics should be used. Through this way, data can be summarized and described; also the degree of variation can be measured. Mean, median and mode are the common measures of central tendency, show the central nature of the data while standard deviation, range, quartiles, interquartile and percentiles are used to present the dispersion of the data. ${ }^{2,5}$ Coefficient of variation is also important measure, useful to compare the variation between/among the groups irrespective of the unit of the variables. If within the variable, variation is minimum, called normal data, but when variation is maximum, considered non-normal data. There are many ways to test the normality of the data, including skewness, kurtosis, standard deviation with resepect to mean, KolmogorovSmirnov test, Shapiro-Wilk test and normality plots. For 
normal data, parametric test is used while non-normal data, nonparametric test is appropriate. ${ }^{4,5}$

Hypothesis is an important step to decide type of statistical technique to be used to test our assumptions. After constructing the hypothesis, use appropriate statistical technique/test to find out whether our hypothesis is true or false and, if true, what is accuracy and strength of the finding. The final result is usually presented in terms of confidence interval and p-value. Minimum 95\% confidence interval or $\mathrm{p}$-value less than 0.05 is considered to be statistically significant. ${ }^{2,5,6}$

When data are continuous scale, and follow normal distribution, parametric test should be used. For comparisons of means between the groups, t-test used while for more than two groups, analysis of variance (ANOVA) (F-test) employed. One sample t-test/z-test is used to test the mean difference between sample with population or given value. Independent $t$-test (between unpaired groups), paired t-test (between two repeated observations of the same group), one way ANOVA (among three or more unpaired groups) and repeated measures ANOVA (among three or more repeated observations of the same group) are other parametric test (Table 1).

When data are continuous, but follow non-normal distribution or in ordinal form, nonparametric test should be used. In nonparametric test, usually the median/ proportion of the groups are compared. For comparison of one group with its population or given value, one sample Wilcoxon signed-rank test should be used. MannWhitney U-test is used to compare between two unpaired groups while between two repeated observations of the same group, related samples Wilcoxon signed-rank test is used. Kruskal-Wallis test is used to compare for more than two unpaired groups while Friedman ANOVA is used to compare among the repeated observations of the same group (Table 1). ${ }^{2,4,5,7,8}$

For categorical data, to test the association between the attributes, Pearson's Chi-square test is used but, for this purpose, each and every cell, expected frequency count should be minimum five. In case of every cell, minimum expected frequency count is minimum five but overall sample size is between 20 and 30, must use Yates continuity correction with Pearson's Chi-square test. When in any cell, minimum expected frequency count is less than five, fisher exact test is more appropriate instead of Pearson's Chi-square test. McNemar Chi-square test is another test in Chi-square family, used to test the effect of intervention between pre- and post-observations when data are dichotomous. Yates continuity correction is also required to be used when total frequency is less than 30 . Cochran's $Q$ test is an extension of McNemar Chi-square test, used to test the effect of intervention among three or more repeated groups. To test the difference in proportions between sample and population/hypothetical value, used one proportion z-test. For comparison between two proportions, when both proportions are from two independent samples, used two proportions z-test (Table 2). 2,6,7

Regression analysis is also a good way to analyze surgical data. For continuous data, linear regression model can be used while for categorical data, logistic regression model used. In linear regression model, when outcome variable is predicted by one independent variable, it is simple linear regression model. In case of more than one independent variable, multivariate linear regression model can be used (Table 1). For linear regression model, dependent variable should follow normal distribution; in case data are not normal, consider log normal, value for both sides to covert data from non-normal to normal, otherwise linear regression can produce wrong prediction. In logistic regression model, binary logistic regression model can be used, when data are nominal and dichotomous scale. If model has one independent variable, it is univariate logistic regression and two or more independent variable called multivariate logistic regression model. For multinominal scale data, multinominal logistic regression model can be used. ${ }^{2,5}$

Table 1: Statistical test for normal and non-normal data

\begin{tabular}{|c|c|c|}
\hline Description & $\begin{array}{l}\text { Parametric test (continuous scale } \\
\text { with normal data) }\end{array}$ & $\begin{array}{l}\text { Nonparametric test (non-normal data or } \\
\text { ordinal scale) }\end{array}$ \\
\hline Descriptive statistics & Mean, standard deviation & Median, interquartiles range \\
\hline $\begin{array}{l}\text { One group (sample) with population } \\
\text { (or hypothetical value) }\end{array}$ & $\begin{array}{l}\text { One sample t-test for small sample } \\
\text { and z-test for large size sample }\end{array}$ & One sample Wilcoxon signed rank test \\
\hline Two unpaired groups & Independent t-test & Mann-Whitney U-test \\
\hline Two paired groups & Paired t-test & Related samples Wilcoxon signed-rank test \\
\hline More than two paired groups & Repeated measures ANOVA & Friedman ANOVA \\
\hline Degree of linear relationship between variables & Pearson's correlation coefficient & Spearman rank correlation coefficient \\
\hline $\begin{array}{l}\text { Predict one outcome variable by one } \\
\text { independent variable }\end{array}$ & Simple linear regression model & $\begin{array}{l}\text { Nonlinear regression model/simple linear } \\
\text { regression model for log normal data }\end{array}$ \\
\hline $\begin{array}{l}\text { Predict one outcome variable by more than one } \\
\text { independent variables }\end{array}$ & Multivariate linear regression model & $\begin{array}{l}\text { Nonlinear regression model/multivariate } \\
\text { linear regression model for } \\
\text { log normal data }\end{array}$ \\
\hline
\end{tabular}


Survival analysis is also an important branch of statistics, frequently used in data analysis where the probability of surviving/dying over period is calculated (Table 3). Life table, Kaplan-Meier and Cox proportion hazard model are frequently used in the study. Life table is useful when probability of surviving is calculated for a group overtime for certain interval. Kaplan-Meier method is used to show the probability of surviving at every event with comparisons between the groups using log rank test. Cox proportional hazard model is a multivariate technique to assess the effect of covariate's over probability of surviving. In survival analysis, measure of risk is presented in terms of hazard ratio. ${ }^{9-11}$

For measurement of accuracy of the diagnostic test, sensitivity (the ability of a test to correctly classify an individual as 'diseased'), specificity (the ability of a test to correctly classify an individual as 'disease-free') and overall accuracy (the ability of a test to correctly classify an individual as 'disease' or 'disease-free') are calculated. Other measures are false positive (1-specificity), false negative (1-sensitivity), likelihood ratio positive (sensitivity/false positive), likelihood ratio negative (false negative/specificity).

Receiver operator characteristic (ROC) curves-usually used to find out-cut off value of the risk parameters with their sensitivity and specificity-are a plot of false positives (1-specificity) against true positives (sensitivity) for all cut-off values. The area under the curve of a perfect test is 1.0 and a useless test is 0.5 . (Table 3). ${ }^{2,12}$

\section{CONCLUSION}

Statistical analysis is an essential component of all biomedical research. To select an appropriate statistical test, it should be clear in one's mind that each method is based

Table 2: Statistical test for categorical data

\begin{tabular}{|c|c|}
\hline Description & Dichotomous \\
\hline Descriptive statistics & Proportion \\
\hline $\begin{array}{l}\text { Comparison between sample } \\
\text { proportion and population proportion }\end{array}$ & One proportion z-test \\
\hline $\begin{array}{l}\text { Comparison of two proportions of } \\
\text { independent samples }\end{array}$ & $\begin{array}{l}\text { Two proportions z-test/ } \\
\text { Chi-square test }\end{array}$ \\
\hline Two paired groups & $\begin{array}{l}\text { McNemar Chi-square } \\
\text { test }\end{array}$ \\
\hline More than two unpaired groups & Chi-square test \\
\hline More than two paired groups & Cochran's Q \\
\hline Association & $\begin{array}{l}\text { Pearson's Chi-square } \\
\text { test/Fisher's exact test }\end{array}$ \\
\hline $\begin{array}{l}\text { Predict one outcome variable by one } \\
\text { independent variable }\end{array}$ & $\begin{array}{l}\text { Univariate logistic } \\
\text { regression model }\end{array}$ \\
\hline $\begin{array}{l}\text { Predict one outcome variable by more } \\
\text { than one independent variables }\end{array}$ & $\begin{array}{l}\text { Multivariate logistic } \\
\text { regression model }\end{array}$ \\
\hline
\end{tabular}

Table 3: Statistical methods for survival data and evaluation of diagnostic test

\begin{tabular}{ll}
\hline Description & Methods \\
\hline $\begin{array}{l}\text { Probability of surviving for equal } \\
\text { intervals }\end{array}$ & Life table \\
$\begin{array}{l}\text { Probability of surviving at every } \\
\text { event with comparisons the groups }\end{array}$ & Kaplan-Meier \\
$\begin{array}{l}\text { Effect of covariate on probability } \\
\text { of surviving }\end{array}$ & Cox proportional hazard \\
$\begin{array}{l}\text { Evaluation of diagnostic test at } \\
\text { different cut-off value }\end{array}$ & ROC curve \\
Accuracy of the diagnostic test & $\begin{array}{l}\text { Sensitivity, specificity, } \\
\text { overall accuracy }\end{array}$ \\
\hline
\end{tabular}

on several underlying assumptions, which have, at least approximately, to be fulfilled in order to obtain correct and meaningful results. Before applying statistical test, important question to be answered includes 'what types of data are being measured in the study?' The test used should be determined by the data. For a correct analysis, researcher has to consider all the possible statistical tests or estimation techniques and their outcomes. To arrive at valid and meaningful conclusion of any study, it is important that all the clinicians reading the literature have sufficient knowledge of statistical methods to facilitate interpretation of increasingly sophisticated statistical analyses.

\section{REFERENCES}

1. Santini L, Conzo G. Endocrine surgery: current and future issues. Int J Surg 2014;12(Suppl 1):S1-2.

2. Sundaram, Karimassery R, Dwivedi SN, Sreenivas V. Medical statistics: principles and methods. Anshan; 2010.

3. Altman, Douglas G. Practical statistics for medical research. CRC Press; 1990.

4. Armitage, Peter, Berry G, Matthews JNS. Statistical methods in medical research. John Wiley \& Sons; 2008.

5. Peat, Jennifer, Barton B. Medical statistics: a guide to data analysis and critical appraisal. John Wiley \& Sons; 2008.

6. Campbell MJ, Machin D. Medical statistics: a commonsense approach. 2nd ed. Chichester: Wiley, Google Scholar 1993.

7. Strasak AM, Zaman Q, Pfeiffer KP, Göbel G, Ulmer H. Statistical errors in medical research: a review of common pitfalls. Swiss Med Wkly 2007 Jan 27;137(3-4):44-49.

8. SPSS, IBM. IBM SPSS statistics base 20. Chicago, IL: SPSS Inc; 2011.

9. Greenhouse, Joel B, Stangl D, Bromberg J. An introduction to survival analysis: statistical methods for analysis of clinical trial data. J Consul Clin Psychol 1989;57(4):536.

10. Bland JM, Altman DG. Survival probabilities (the KaplanMeier method). BMJ 1998 Dec 5;317(7172):1572.

11. Moosazadeh M, Bahrampour A, Nasehi M, Khanjani N. Survival and predictors of death after successful treatment among smear positive tuberculosis: a cohort study. Int J Preventive Med 2014;5(8):1005-1012.

12. Bewick V, Cheek L, Ball J. Statistics review 13: receiver operating characteristic curves. Crit Care 2004 Dec;8(6):508-512. 